\title{
Computing Histogram of Tensor Images using Orthogonal Series Density Estimation and Riemannian Metrics
}

\author{
Emmanuel Chevallier ${ }^{a}$, Augustin Chevallier $^{b}$, Jesús Angulo $^{a}$ \\ ${ }^{a}$ CMM-Centre de Morphologie Mathématique, MINES ParisTech; France \\ ${ }^{b}$ Ecole Normale Supérieur de Cachan; France \\ emmanuel . chevallier@mines-paristech.fr
}

October 2013

\begin{abstract}
This paper deals with the computation of the histogram of tensor images, that is, images where at each pixel is given a $n \times n$ positive definite symmetric matrix, $\operatorname{SPD}(n)$. An approach based on orthogonal series density estimation is introduced, which is particularly useful for the case of measures based on Riemannian metrics. By considering $\operatorname{SPD}(n)$ as the space of the covariance matrices of multivariate gaussian distributions, we obtain the corresponding density estimation for the measure of both the Fisher metric and the Wasserstein metric. Experimental results on the application of such histogram estimation to DTI image segmentation, texture segmentation and texture recognition are included.
\end{abstract}

Keywords: image histogram, density estimation, volume measure of Riemannian metrics, symmetric-positive-definite matrices

\section{Introduction}

The histogram computation of a scalar image consists in counting the number of pixels at each different intensity value. This extremely simple approximation to the univariate density distribution of image intensities is one of the most important image processing tools to address problems such as contrast enhancement (by histogram linear stretching or using advanced 
approaches [15]), image segmentation (by 1D clustering), texture processing [13], image retrieval [5], etc. As digital images underlay a quantization step, a more reliable estimation of the image distribution requires techniques such as the classical Parzen window method or a variational approach as the recently proposed in [10]. Computation of histogram of a color image involves in addition the choice of the color space and an appropriate discretization of the colors in the image into a number of bins.

In this paper we are interested in the computation of the histogram of a tensor image, that is, an image where at pixel we have a value in $\operatorname{SPD}(n)$ : the space of $n \times n$ positive definite symmetric matrices. Recently there has been growing interest on processing methods of such images in the computer vision community [6]. As far as we know, the construction of histogram in $\operatorname{SPD}(n)$-valued images has not been previously addressed. It can be noted that if a normal law density on tensors is assumed, the estimation of the density involves only the computation of the mean tensor as well as a covariance matrix $[9,11]$. In our case, we do not assume that the histogram lays in any specific density family. Straightforward approach based on a discretization of $\operatorname{SPD}(n)$ into bins, followed by a counting of the tensors belonging to each bin, leads to a histogram estimation which suffers from various problems. A better solution involves the use of the Parzen window technique. We adopt here an alternative approach based on orthogonal series density estimation. An important property of this approach is that it depends on geometry only through the volume element, and not on the geodesic distances. The rest of the paper is organized as follows. The precise definition of image histogram adopted in this paper is given in Section 2. Section 3 introduces the notion of orthogonal series density estimation, including the measure change. A brief remind on $\operatorname{SPD}(n)$ space, followed by a discussion on the various measures of $\operatorname{SPD}(n)$ are given in Section 4. In particular, by considering $\operatorname{SPD}(n)$ as the space of the covariance matrices of multivariate gaussian distribution, we obtain the corresponding density estimation for the measure of the Fisher metric and the Wasserstein metric. Section 5 summarizes the framework for estimating the histograms of tensor valued images and some experimental results on the application of such histogram estimation to DTI image segmentation, texture segmentation and texture recognition. Conclusions in Section 6 end the paper.

\section{Image histogram}

In this section we set the theoretical link and notation between images and probabilities. We assimilate the notion of image histogram and the notion 
of density estimation. Let us consider an image $I$ as the map:

$$
I:\left\{\begin{array}{ccc}
\Omega & \rightarrow & V \\
p & \mapsto & I(p)
\end{array}\right.
$$

We have for instance $V=\mathbb{R}$ for grey-scale images or $V=\mathbb{R}^{n}$ for multispectral images; $\Omega$ is the support space of pixels/voxels, typically a subset of $\mathbb{R}^{2}$ or $\mathbb{R}^{3}$ such as a rectangle or a parallelepiped. In the case of tensor images, which is the case addressed in this paper, $V \subseteq \operatorname{SPD}(n)$.

We endow $\Omega$ with a measure $P$ that reflects its geometry: as the spatial distances are usually evaluated using Euclidean distance, this measure is typically the Lebesgue measure. Indeed the prior importance of an area is often proportional to its Lebesgue measure. After rescaling the measure, the map $I$ can be seen as a random variable. The law of the random variable $I$ contains a synthetic information of the image. Thus, one might be interested in the study of this law. We have access to a finite number of evaluation of $I$ in points $p \in \Omega$ called pixels. Each $I(p)$ is an evaluation of the random variable $I$. The set $\{I(p), p$ a pixel $\}$ is a set of draws of $I$. We will assume that the set of pixel is uniformly distributed according to the probability $P$. Formally, if $\phi: \Omega \rightarrow \mathbb{R}$ is a continuous function, $\frac{1}{N} \sum_{p} \phi(p) \rightarrow \int \phi d P$ when the number of pixel $N$ tends to infinity. If they are not uniformly distributed, we have to take their distribution into account to estimate the law of $I$. This for instance the case for point cloud images [4].

The study of law of $I$ is made through this set of draws. We assume that a measure $\mu$ is given on the space $V$. Furthermore, we make the strong assumption that the law of $I$ has a density with respect to $\mu$. The problem now is to estimate this density from the sample set $\{I(p), p$ a pixel $\}$. The classical way to proceed is to cut the space $V$ into regular bins and to count the number of draws in each bin. The precision of the estimation depends on the measure of the bins, and on the number of draws. However under some particular circumstances it might be impossible to get such a cutting of the space $V$ in regular bins of same measure. This is often the case is $\mu$ is not the Lebesgue measure. If the measures of the bins are not equal, problems appear when the set of draws is not large enough. Let us consider for instance two adjacent bins $b_{1}$ and $b_{2}$ with $\mu\left(b_{1}\right)$ much bigger than $\mu\left(b_{2}\right)$. If a draw falls in $b_{2}$, the obtained density will be much higher than if the draw had fallen in $b_{1}$. This example shows that a small variation in the draw can induce important variations on the values taken by the estimated density. 


\section{Orthogonal series density estimation}

Let us assume that $V$ is a compact subset of $\mathbb{R}^{n}$, with the measure $\mu$. The measure $\mu$ defines a scalar product on the set $L^{2}(\mu)$ of square integrable functions given by $\langle f, g\rangle=\int f g d \mu$. Let us also assume that $\left\{e_{i}\right\}$ is a Hilbert basis of the functions from $V$ to $\mathbb{R}$, for the previous scalar product. The density of $I$ against the measure $\mu$, denoted $f_{I}$, can be studied through its expression in this generator system. Indeed we have

$$
f_{I}=\sum_{i=-\infty}^{\infty}\left\langle f_{I}, e_{i}\right\rangle e_{i}
$$

where

$$
\left\langle f_{I}, e_{i}\right\rangle=\int f_{I} e_{i} d \mu=\mathbb{E}\left(e_{i}(I)\right) .
$$

Thus $\left\langle f_{I}, e_{i}\right\rangle$ can easily be approximated by an estimation of the expectation, i.e.,

$$
\mathbb{E}\left(e_{i}(I)\right) \approx \frac{1}{n} \sum_{j=1}^{n} e_{i}\left(I\left(p_{j}\right)\right)
$$

where $n$ is the number of pixels of image $I$. We have then

$$
f_{I} \approx \sum_{i=-\infty}^{\infty}\left[\frac{1}{n} \sum_{j=1}^{n} e_{i}\left(I\left(p_{j}\right)\right)\right] e_{i},
$$

or in the case of a finite number of basis functions:

$$
f_{I} \approx \sum_{i=-N}^{N}\left[\frac{1}{n} \sum_{j=1}^{n} e_{i}\left(I\left(p_{j}\right)\right)\right] e_{i}=\hat{f}_{I} .
$$

Thus we consider $\hat{f}_{I}$ as an estimate of the density $f_{I}$ from the $I\left(p_{j}\right)$. We note that using this method, if the $\left\{e_{i}\right\}$ are continuous functions then the estimation is continuous according to the $I\left(p_{j}\right)$. This was not the case using the cutting in bins described previously.

The estimation can be easily adapted to a new measure $\mu^{\prime}$ if it has a density $f_{\mu^{\prime}}$ with respect to $\mu$. The new density $f_{I}^{\prime}$ becomes

$$
f_{I}^{\prime}=\frac{f_{I}}{f_{\mu^{\prime}}}
$$

Relationship (2) can be easily obtained using the new orthonormal basis system $\left\{e_{i} / \sqrt{f_{\mu^{\prime}}}\right\}$ instead of $\left\{e_{i}\right\}$. Indeed we have

$$
\int \frac{e_{i}}{\sqrt{f_{\mu^{\prime}}}} \frac{e_{j}}{\sqrt{f_{\mu^{\prime}}}} d \mu^{\prime}=\int e_{i} e_{j} d \mu
$$


Hence, the new system is orthonormal for $\mu^{\prime}$ and consequently, we have

$$
\begin{aligned}
\hat{f}_{I}^{\prime} & =\sum_{i=-N}^{N}\left[\frac{1}{n} \sum_{j=1}^{n} \frac{e_{i}\left(I\left(p_{j}\right)\right)}{\sqrt{f_{\mu^{\prime}}}}\right] \frac{e_{i}}{\sqrt{f_{\mu^{\prime}}}} \\
& =\frac{\sum_{i=-N}^{N}\left[\frac{1}{n} \sum_{j=1}^{n} e_{i}\left(I\left(p_{j}\right)\right)\right] e_{i}}{f_{\mu^{\prime}}}=\frac{\hat{f}_{I}}{f_{\mu^{\prime}}} .
\end{aligned}
$$

This property will prove very useful for our work. A deeper presentation of density estimation using orthogonal series can be found in [3].

\section{The space $\operatorname{SPD}(n)$ and its parametrization}

A differentiable manifold is a topological space where the local neighbourhood of each point looks like a vector space. In each point of a manifold we can associate a tangent space composed of tangent vectors to all smooth curves going through the considered point. One can introduce a notion of distance on a manifold by defining a scalar product on each tangent space. If the different scalar product are compatible, that is to say vary smoothly between tangent space, it is possible to define a notion of length of a curve on the manifold. Such a $\mathrm{m}$ anifold is called a Riemannian manifold.

We will set here a few notations and describe the structure of the space $\operatorname{SPD}(n)$. Let $\mathrm{M}(n)$ represents the space of $n \times n$ square matrices and $\mathrm{GL}(n)$ the subset of $\mathrm{M}(n)$ of invertible matrices. By $\operatorname{Sym}(n)$, one denotes the space of symmetric matrices. Let $A$ be a matrix in $\operatorname{Sym}(n) . A$ is positive semidefinite if $\forall x \in \mathbb{R}^{n}, x^{t} A x \geq 0$ where $x^{t}$ denotes the transpose of $x$. The set of such matrices $A$ forms a close convex cone whose interior is called the set of symmetric positive definite matrices and is noted $\operatorname{SPD}(n)$. If $A$ is in the interior of the cone then we have $x^{t} A x>0$. A complete description of the $\operatorname{SPD}(n)$ space can be found in [2]. Equipped with different scalar products, $\operatorname{SPD}(n)$ can be seen as a Riemannian manifold.

\subsection{Two parametrisations of $\operatorname{SPD}(n)$}

In order to compute the histogram of an image valued in $\operatorname{SPD}(n)$ matrices, one needs to set the parametrization framework of this space. Indeed there are many different ways to study $\operatorname{SPD}(n)$ matrices. The $\operatorname{SPD}(n)$ matrices can be seen at first as a subset included in the space $\mathrm{M}(n)$. However, this framework is not adapted to histogram computation for the following reason. The random variable $I$ is valued in $\operatorname{SPD}(n)$ only. Now, as a vector subspace, the space $\operatorname{SPD}(n)$ has a null measure according to the Lebesgue measure of 
matrices. Thus the random variable $I$ cannot have a density against the Lebesgue measure of matrices $\mathrm{M}(n)$.

A way to overcome this problem is to study the $\operatorname{SPD}(n)$ space as a subset of the $\operatorname{Sym}(n)$ matrices. In that case the $\operatorname{space} \operatorname{SPD}(n)$ has a non null measure according to the Lebesgue measure of $\operatorname{Sym}(n)$. Each matrix can be expressed in the canonical basis of symmetrical matrices, coordinates forms what we will call parametrization 1 .

A second approach involves decomposing $\operatorname{SPD}(n)$ matrices over rotations and eigenvalues. A symmetrical matrix can be diagonalized in an orthonormal basis. Then each $\operatorname{SPD}(n)$ matrix can be represented by its eigenvalues and a rotation matrix. Using any angular representation of the rotation matrix, the $\operatorname{SPD}(n)$ matrix can be represented by a set of eigenvalues and a set of angles. This representation forms a convex subset of non null measure of $\mathbb{R}^{\frac{n(n+1)}{2}}$. We will call this parametrization 2 .

Now comes the question of the reference measure $\mu$ used to compute the histogram. To each parametrization of the $\operatorname{SPD}(n)$ space in $\mathbb{R}^{\frac{n(n+1)}{2}}$ is associated a Lebesgue measure. One must be aware that the Lebesgue measure of each parametrization is different. Thus, given two different parametrizations, the computation of histograms against their Lebesgue measure will give different results. Besides Lebesgue measures, other measures can be computed from the two main Riemannian metrics on $\operatorname{SPD}(n)$ : the Fisher metric and the Wasserstein metric. Indeed any Riemannian metric gives place to a volume measure.

We explain now a key property of the parametrization 2. Given any measure on $\operatorname{SPD}(n)$ which is invariant by the action of the rotation, i.e., $(M, R) \mapsto R M R^{t}$, the measure is a product between a measure on the eigenvalues and a the Haar measure of the rotation. Indeed, for all such measures on parametrization 2, it enables us to separate the study of the eigenvalues and the rotations. This result can be found in [18], but a for the convenience of the reader, a simple proof is given in Appendix B.

As we explained previously, the change of coordinates between both parametrizations induces a change in volume measure. The modification of the density of the volume measure is expressed by the Jacobian of the change of coordinates [8]:

$$
\begin{aligned}
\phi: \mathbb{R}^{\frac{n(n+1)}{2}} & \rightarrow \operatorname{SPD}(n) \\
\left(\lambda_{1}, \cdots, \lambda_{n}, \theta_{1}, \cdots, \theta_{\frac{n(n-1)}{2}}\right) & \mapsto R_{\theta}\left(\begin{array}{ccc}
\lambda_{1} & 0 & \cdots \\
0 & \cdots & \\
\cdots & & \lambda_{n}
\end{array}\right) R_{\theta}^{t}
\end{aligned}
$$

where $\left\{\lambda_{i}\right\}$ are the $n$ positive eigenvalues ordered in increasing ordering and 
$\left\{\theta_{j}\right\}$ are the $n(n-1) / 2$ angles which parametrize the rotation matrix $R_{\theta}$.

Let us write $\mu_{\text {Lebes }_{1}}$ the Lebesgue measure of the parametrization 1 of $\operatorname{SPD}(n)$ and $\mu_{\text {Lebes }_{2}}$ the Lebesgue measure of parametrization 2. The computation of the Jacobian of the coordinate change gives the following relation $[8]$ :

$$
\frac{d \mu_{\text {Lebes }_{1}}}{d \mu_{\text {Lebes }_{2}}}=\left(\prod_{1 \leq i<j \leq n}\left|\lambda_{i}-\lambda_{j}\right|\right) h_{\mathrm{Haar}}\left(\theta_{1}, \ldots, \theta_{\frac{n(n-1)}{2}}\right)
$$

where $h_{\text {Haar }}\left(\theta_{1}, \ldots, \theta_{\frac{n(n-1)}{2}}\right)$ is the density of the Haar measure on rotation in the parametrization ${ }^{2}$. In the following it will be denoted by $h_{\text {Haar }}$.

\subsection{Measure for the Fisher and the Wasserstein metrics}

We are interested now in the expression of the density of the volume measure of the Fisher metric and the Wasserstein metric in both parametrizations. The Fisher metric on $\operatorname{SPD}(n)$ is the metric induced by the geometry of information [1]. This metric is invariant under the following action of $\operatorname{Gl}(n)$ on $\operatorname{SPD}(n)$, i.e.,

$$
\begin{aligned}
& a: \operatorname{Gl}(n) \times \operatorname{SPD}(n) \rightarrow \operatorname{SPD}(n) \\
& (G, M) \mapsto G M G^{t}
\end{aligned}
$$

The Wasserstein metric is another well known metric on $\operatorname{SPD}(n)$ induced by the optimal transport of centered Gaussian laws [17]. Given a Riemannian metric, the density of the volume measure is given by $\sqrt{\operatorname{det}(G)}$, where $G$ is the matrix of the metric [11]. Given two symmetric matrices $U, V \in \operatorname{Sym}(n)$ and a matrix $W \in \operatorname{SPD}(n)$, the scalar product between $U$ and $V$ at the $W$ matrix, take respectively the following forms for the Fisher [12] and the Wasserstein [17] metric:

$$
\begin{aligned}
\langle U, V\rangle_{W}^{\text {Fisher }} & =\operatorname{tr}\left(W^{-\frac{1}{2}} U W^{-1} V W^{-\frac{1}{2}}\right), \\
\langle U, V\rangle_{W}^{\text {Wasserstein }} & =\operatorname{tr}(U W V) .
\end{aligned}
$$

From these scalar products we can compute the matrices of the metrics $G_{W}^{\text {Fisher }}$ and $G_{W}^{\text {Wasserstein }}$. Then if we express $G$ in the canonical base of symmetric matrices we have:

$$
\begin{aligned}
\sqrt{\operatorname{det}\left(G_{W}^{\text {Fisher }}\right)} & =\alpha \sqrt{\prod_{1 \leq i \leq n} \frac{1}{\lambda_{i}^{n+1}}}, \\
\sqrt{\operatorname{det}\left(G_{W}^{\text {Wasserstein }}\right)} & =\alpha \sqrt{\prod_{1 \leq i \leq n} \lambda_{i} \prod_{1 \leq i<j \leq n}\left(\lambda_{i}+\lambda_{j}\right)},
\end{aligned}
$$


where $\left\{\lambda_{i}\right\}$ are the eigenvalues of $W$ and $\alpha$ is a multiplicative constant which depends on the dimensionality of the space, i.e $\alpha=\frac{1}{2^{\frac{n(n-1)}{2}}}$. These expressions represent the densities of the Fisher and Wasserstein measures with respect to the Lebesgue measure of symmetric matrices $\mu_{\text {Lebes }_{1}}$.

Using the expression (4) of the density of the Lebesgue measure of symmetric matrices against the Lebesgue measure of the parametrisation 2, we obtain the expressions of the density of the Fisher and Wasserstein measures against the Lebesgue measure of the $\mu_{\text {Lebes }_{2}}$, given respectively by:

$$
\begin{gathered}
\frac{d \mu_{\text {Fisher }}}{d \mu_{\text {Lebes } 2}}=\alpha \frac{\prod_{1 \leq i<j \leq n}\left|\lambda_{i}-\lambda_{j}\right|}{\sqrt{\prod_{1 \leq i \leq n} \lambda_{i}^{n+1}}} h_{\text {Haar }}, \\
\frac{d \mu_{\text {Wasserstein }_{1}}}{d \mu_{\text {Lebes }_{2}}}=\alpha \sqrt{\prod_{1 \leq i \leq n} \lambda_{i} \prod_{1 \leq i<j \leq n}\left(\lambda_{i}+\lambda_{j}\right)}\left(\prod_{1 \leq i<j \leq n}\left|\lambda_{i}-\lambda_{j}\right|\right) h_{\text {Haar }} .
\end{gathered}
$$

Despite the fact that we did not find the expression (5) in the literature, this formula might already be known. However, we believe that the case of the density of Wasserstein measure against the Lebesgue has not been previously published elsewhere. Detailed derivations are given in Appendix A.

\section{Experimental results on tensor image histograms}

Given a tensor image $I: \Omega \rightarrow \operatorname{SPD}(n)$, we perform one of the previous changes of coordinates for each pixel value, that is to say we represent each matrix in one of the parametrizations. The Fourier basis associated with each parametrization forms an orthonormal system with respect to the Lebesgue measure. According to methodology from Section 3, density estimate $\hat{f}_{I}$ can be evaluated against this basis. The choice of the Fourier basis gives place to an efficient estimation of the image density against the Lebesgue measure of the parametrisation 2 associated to decoupling eigenvalues and rotation angles. We obtain then the histogram of the tensor image according to the Lebesgue measure of the parametrisation 2, i.e., $\hat{f}_{I ; \mu_{\text {Lebes }} 2}$. As explained in Section 3 , it is possible to adapt the histogram to any other measure that has a density against the Lebesgue measure by the expression (3). For instance, the histogram estimate according to the Fisher metric measure is obtained by means of (5) by

$$
\hat{f}_{I ; \mu_{\text {Fisher }}}=\hat{f}_{I ; \mu_{\text {Lebes } 2}} \frac{d \mu_{\text {Fisher }}}{d \mu_{\text {Lebes }_{2}}} .
$$


Obviously, after choosing the family of the orthogonal functions of the basis, we need to fix the number of components. For the experiments of this paper we have used the basis:

$$
\left\{e_{-i}(x)=\sqrt{2} \sin (i \pi x) ; e_{0}(x)=1 ; e_{i}(x)=\sqrt{2} \cos (i \pi x) ; i=1, \cdots, N\right\}
$$

We now present applications of histogram construction for tensor images. All what follows is achieved according to the framework described previously. For computational reasons the applications on $\operatorname{SPD}(n)$ are here limited to low dimensions, namely $n=2$ for structure tensors from 2D texture images, and $n=3$ for diffusion tensor images (DTI).

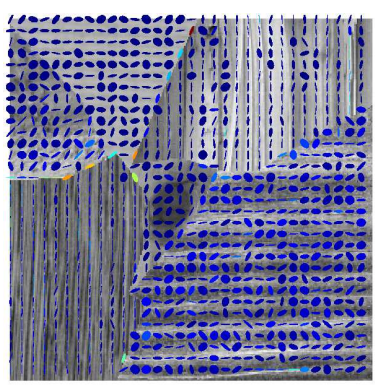

(a)

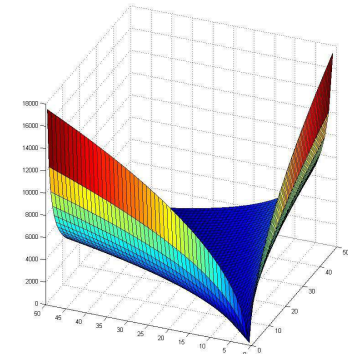

(c)

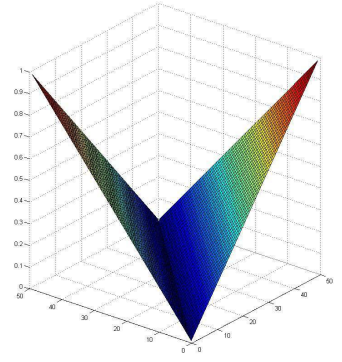

(b)

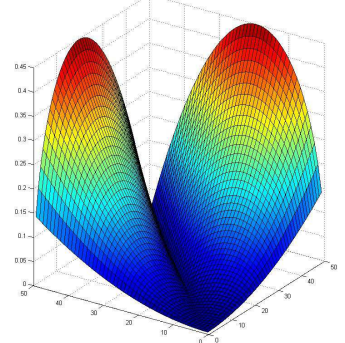

(b)

Figure 1: Image (a) is an example of structure tensor image $I$. (b), (c),(d) represent respectively the densities of $\mu_{\text {Lebes }_{1}}, \mu_{\text {Fisher }}$ and $\mu_{\text {Wasserstein }}$ with respect to $\mu_{\text {Lebes }_{2}}$ as a function of the eigenvalues.

\subsection{Image segmentation}

The first application consists in segmenting tensor images using their histograms. We first compute the complement histogram of the $\operatorname{SPD}(n)$ image and then apply a watershed transform [16] on the histogram. We note that 
the histogram can be view as a $n(n+1) / 2$-dimensional grey-level image and the corresponding watershed transformation will provide a partition of $I\left(\{\Omega\}\right.$ in connected sets $A_{i}: I(\Omega)=\cup A_{i}$. In particular, each class $i$ is associated to a regional maximum of the histogram. The segmentation of the image is obtained by assigning a class $i$ to each pixel according to its class on the segmented histogram: $\Omega=\cup I^{-1}\left(A_{i}\right)$

We consider $2 \mathrm{D}$ grey-scale texture segmentation using the classical structure tensor representation [7]. More precisely, given an image composed of different textures, we calculate the structure tensor at each pixel, using a window size that we expect, makes the structure tensor as constant as possible on each texture class of the image. At this point, we have an image where each pixel is a point of $\operatorname{SPD}(2)$, parametrized by two eigenvalues and one angle. Then the 3D histogram is computed with respect to one the considered metrics. Fig. 1 shows an example of structure tensor image and its histograms using different measures $\mu_{\text {Lebes }_{2}}, \mu_{\text {Fisher }}$ and $\mu_{\text {Wasserstein. }}$. We note that for visualization purposes we have only considered the histogram of the two eigenvalues. It should be also remarked that the contribution concerning the angle by measure $h_{\text {Haar }}$ is independent of the metric.

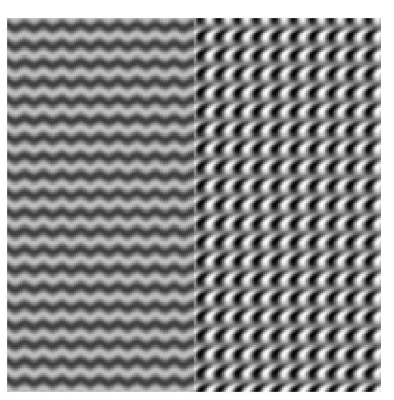

(a)

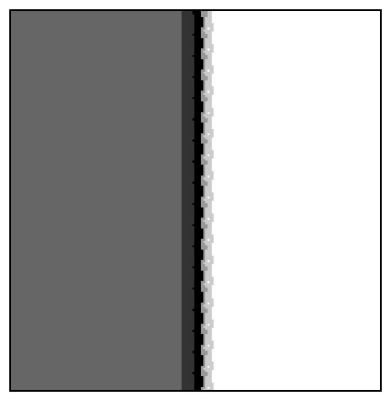

(b)

Figure 2: Image texture segmentation by watershed partitioning of structure tensor histogram using Wasserstein metric: (a) original image; (b) segmentation.

Fig. 3 shows the segmentation obtained for a synthetic texture image. As expected, homogenous texture areas are well separated, but the "texture contours" introduce additional texture classes. In particular, this example corresponds to the result for the Wasserstein metric, similar ones are obtained for the other measures. Results given in Fig. 3 correspond to a more complex texture with a comparison of two metrics. From our experiments, we cannot conclude if one of the metrics is better than another for the purpose of texture segmentation. In addition, the obtained segmenta- 
tion depends also on the regularization window from the structure tensor computation.

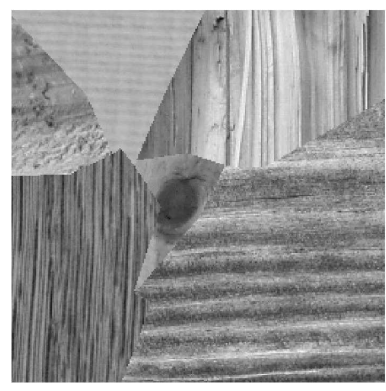

(a)

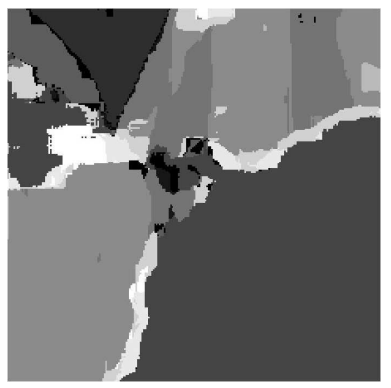

(b)

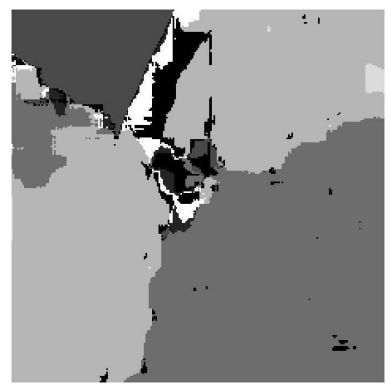

(c)

Figure 3: Image texture segmentation by watershed partitioning of structure tensor histogram: (a) original image; (b) segmentation using metric $\mu_{\text {Lebes2 }}$; (c) segmentation using metric $\mu_{\text {Wasserstein }}$.

The second case study deals with the segmentation of DTI images, thus $I(\Omega) \subseteq \operatorname{SPD}(n)$, a 6-dimensional manifold where each matrix is represented by three eigenvalues and three angles. We can apply the same method as the one we used for tensor structure segmentation. An example of DTI segmentation is shown in Fig. 4. We have compared the various metrics. We have observed for instance that for a global segmentation of the brain into its main areas, the best segmentation results are obtained using only the dominant eigenvalue with respect to its Lebesgue measure, see Fig. 4(b). For this example, we have compared also the performance of segmentation using a histogram obtained by discretization into bins and counting, see Fig. 4(c). As we can observe, this histogram includes an important number of peaks which produces a strong over segmentation into many classes.

\subsection{Texture recognition}

In our last application, we use the structure tensor histogram in order to perform texture recognition. We based ourselves on the Brodatz database [14] that contains 111 texture images. Each image of the database has been vertically cut into two equal parts. This way, we built a test set and reference set. For each image of the test and the reference set, we computed the histogram of structure tensor. Structure tensor images are computed for different size $s$ of averaging window. For each histogram of the test set, we perform a comparison with all the histograms of the reference set, and select the closest one. The matching is correct if the selected histogram corresponds to the other other half of the image in the reference set. 


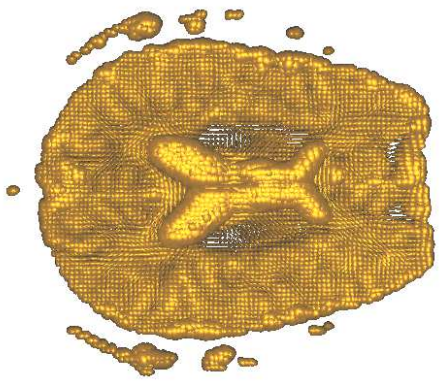

(a)

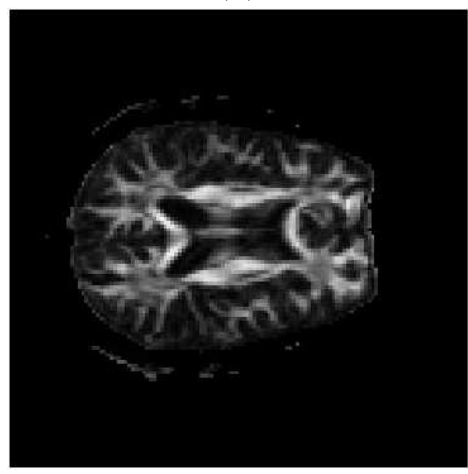

(b)

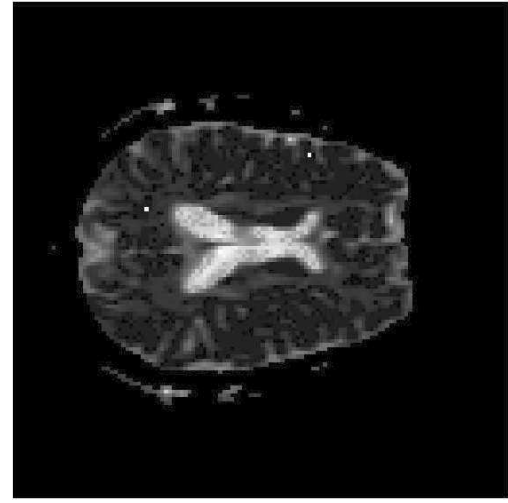

(c)

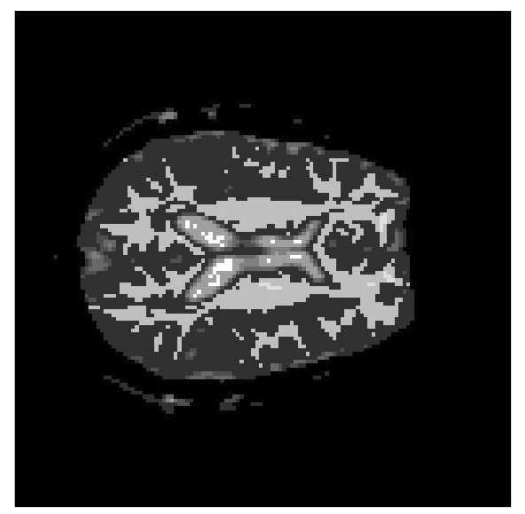

Figure 4: DTI segmentation by watershed partitioning of its histogram: (a) original image; (b) fractional anisotropy; (c) segmentation using a histogram obtained by discretization into bins and counting; (d) segmentation using orthogonal series-based histogram.

The histogram based on bins gives interesting results in term of performance of classification. However, results are significantly different when using parametrisation 1 or parametrization 2 . Indeed the size and the shape of the bins are totally different in the two parametrizations, and we obtain two different estimations of the density. Thus we based the experiment on orthogonal series. The comparison between histograms can be achieved according to several norms. Despite the fact that the $L_{2}$ norm might no be the best measure between histograms, it presents a serious advantage in our case. Let $c_{i}$ denote $\left\langle f, e_{i}\right\rangle$. An approximation of the $L_{2}$ norm can be obtained directly from the coefficients $\hat{c}_{i}=\frac{1}{n} \sum_{j=1}^{n} e_{i}\left(I\left(p_{j}\right)\right)$ without performing any discretization of the density $f$. Let $c_{i}^{1}$ and $c_{i}^{2}$ be respectively the coefficient associated with the densities $f_{I_{1}}$ and $f_{I_{2}}$. The $L_{2}$ norm between these two 


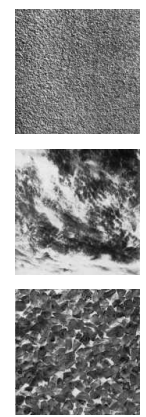

\begin{tabular}{|c|c|c|c|c|}
\hline & $\mu_{\text {Wasserstein }}$ & $\mu_{\text {Lebes }_{1}}$ & $\mu_{\text {Lebes } 2}$ & $\mu_{\text {Fisher }}$ \\
\hline$s=3$ & 39 & 72 & 90 & 89 \\
\hline$s=11$ & 67 & 78 & 85 & 88 \\
\hline$s=21$ & 72 & 78 & 78 & 80 \\
\hline$s=41$ & 71 & 72 & 67 & 72 \\
\hline
\end{tabular}

(b)

(a)

Table 1: (a) Three examples from the Brodatz texture dataset. (b) Accuracy (in percentage) of texture recognition in Brodatz database using structure tensor histogram with respect to various measures. Parameter $s$ denotes the size of the window used in structure tensor estimation.

densities can be approximated by

$$
\left\|f_{I_{1}}-f_{I_{2}}\right\|_{L_{2}}^{2} \approx \sum_{i=-N}^{N}\left(c_{i}^{I_{1}}-c_{i}^{I_{2}}\right)^{2} .
$$

Other norms or divergences requires a preliminary discretization. Results of Brodatz texture recognition are summarized in Table 1. A possible conclusion from this comparison is the stability of relevant results for Fisher metric against the size parameter of the structure tensor.

\section{Conclusion}

We started to explore different methods to address the construction of histograms for tensor images. The standard way to build histograms using bins has the advantage of a low computational cost. However, as previously explained, and as different applications show, this method might not always be the most adapted, mainly due to the irregularity induced by the size of the bins. Orthogonal series proved to be an interesting alternative. The choice of the parametrization has also been addressed. Both parametrization 1 and parametrization 2 can be chosen to study histograms. However, parametrization 2 has the following important property : a measure on $\mathrm{SPD}(\mathrm{n})$ invariant by the action of rotation $R M R^{t}$ is a product measure between a measure on eigenvalues and a measure on angles. On the other hand, the choice of the orthogonal basis still remains fully open. We chose to use Fourier basis of the different parametrization due to their simplicity. However, other basis might be more adapted to the different measures, in 
terms of regularity. Good candidates are for instance the eigenfunctions of the Laplacian operator associated to the different metrics.

\section{References}

[1] S. Amari and H. Nagaoka. Methods of information geometry. AMS and Oxford university press, 2000.

[2] R. Bhatia. Positive Definite Matrices. Princeton University, 2007.

[3] S. Efromovich. Orthogonal series density estimation. Wiley Interdisciplinary Reviews: Computational statistics, 2:467-476, 2010.

[4] Steve Oudot Frdric Chazal, Leonidas J. Guibas and Primoz Skraba. Scalar field analysis over point cloud data. Discrete and Computational Geometry, 46(4):743-775, 2011.

[5] Y. Gong, C.H. Chuan, and G. Xiaoyi. Image indexing and retrieval using color histograms. Multimedia Tools and Application, 2:133 - 156, 1996.

[6] S. Jayasumana, R. Hartley, M. Salzmann, H. Li, and M. Harandi. Kernel methods on the riemannian manifold of symmetric positive definite matrices. In Proceedings of CVPR'13, 2013.

[7] H. Knutsson. Representing local structure using tensors. In Oulu University, editor, Proceedings of 6th Scandinavian Conf. on Image Analysis, page 244 251, 1989.

[8] S. Kuriki and A. Takemura. Some geometry of the cone of the nonnegative definite matrices and weight of associated $\chi^{2}$ distribution. Ann. Inst. Statist. Math., 52(1):1-14, 2000.

[9] C. Lenglet, M. Rousson, R. Deriche, and O. Faugeras. Statistics on the manifold of multivariate normal distributions: Theory and application to diffusion tensor mri processing. Journal of Mathematical Imaging and Vision, 25(3):423-444, 2006.

[10] M. Nikolova, Y.-W. Wen, and R. Chan. Exact histogram specification for digital images using a variational approach. Journal of Mathematical Imaging and Vision, 46(3):309 - 325, 2013.

[11] X. Pennec. Intrinsic statistics on riemannian manifolds: Basic tools for geometric measurements. Journal of Mathematical Imaging and Vision, 25(1):127-154, 2006. 
[12] X. Pennec, P. Fillard, and N. Ayache. A riemannian framework for tensor computing. International Journal of Computer Vision, 66(1):4166, 2006.

[13] J. Portilla and E.P. Simoncelli. A parametric texture model based on joint statistics of complex wavelet coefficients. International Journal of Computer Vision, 40:49 71, 2000.

[14] T. Randen and J.H. Husøy. Filtering for texture classification: A comparative study. IEEE Trans. Pattern Analysis and Machine Intelligence, 21(4):291 310, 1999.

[15] G. Sapiro and V. Caselles. Histogram modification via differential equations. Journal of Differential Equations, 135(2):238 - 268, 1997.

[16] P. Soille. Morphological Image Analysis: Principles and Applications. Springer-Verlag New York, 2 edition, 2003.

[17] A. Takatsu. Wasserstein geometry of gaussian measures. Osaka J. Math, 48:1005-1026, 2010.

[18] Robert A Wijsman. Invariant measures on groups and their use in statistics. Institute of Mathematical Statistics, Hayward, 1990.

\section{A Density of Fisher and Wasserstein volume mea- sure}

Let us start by considering

$$
\begin{gathered}
\langle U, V\rangle_{W}^{f i s h e r}=\operatorname{tr}\left(W^{-\frac{1}{2}} U W^{-1} V W^{-\frac{1}{2}}\right) \\
\langle U, V\rangle_{W}^{W a s s e r s t e i n}=\operatorname{tr}(U W V)
\end{gathered}
$$

Let $E_{i, j}$ be the canonical basis of $M(n)$. Let $E_{i, i}^{\prime}=E_{i, i}$ and $E_{i, j}^{\prime}=$ $\frac{E_{i, j}+E_{j, i}}{\sqrt{2}}$ if $i<j$ The set of matrices $E_{i, j}^{\prime}, i \leq j$, forms a basis of the symmetric matrices. This basis is orthonormal for the Euclidean scalar product on matrices. We can notice that for any rotation $R$ the basis formed by the $R E^{\prime}{ }_{i, j} R^{-1}$ is also orthogonal for the Euclidean scalar product.

Let $G_{W}$ be the matrix of the metric at a given $W=R D R^{-1}$, expressed in an orthonormal basis for the euclidean scalar product. The positive number $\sqrt{\operatorname{det}\left(G_{W}\right)}$ represents the volume measure associated to the metric $G$ with respect to the volume measure associated to the euclidean scalar product. 
We are interested in the calculation of $\sqrt{\operatorname{det}\left(G_{W}\right)}$ for the Fisher and the Wasserstein metrics.

Let us start with the Fisher metric. We have

$$
\left\langle R E^{\prime}{ }_{i, i} R^{-1}, R E^{\prime}{ }_{j, j} R^{-1}\right\rangle_{W}^{f i s h e r}=\frac{\delta_{i, j}}{\lambda_{i}^{2}},
$$

and for $i \neq j$ and $k \neq l$ :

$$
\left\langle R E^{\prime}{ }_{i, j} R^{-1}, R E^{\prime}{ }_{k, l} R^{-1}\right\rangle_{W}^{f i s h e r}=\frac{\delta_{i, k} \delta_{j, l}}{2 \lambda_{i} \lambda_{j}},
$$

finally for $j \neq k$ :

$$
\left\langle R E_{i, i}^{\prime} R^{-1}, R E^{\prime}{ }_{j, k} R^{-1}\right\rangle_{W}^{f i s h e r}=0,
$$

We can now write the matrix $G_{W}^{F i s h e r}$ in the basis formed by the $R E^{\prime}{ }_{i, j} R^{-1}$.

$$
G_{W}^{\text {Fisher }}=\left(\begin{array}{cccc}
\frac{1}{\lambda_{1}^{2}} & 0 & \cdot & \\
0 & \cdot & & \\
\cdot & & \frac{1}{\lambda_{n}^{2}} & \\
& & & \frac{1}{2 \lambda_{i} \lambda_{j}}
\end{array}\right)
$$

The expression of the volume measure is then obtained as

$$
\sqrt{\operatorname{det}\left(G_{W}^{\text {Fisher }}\right)}=\frac{1}{2^{\frac{n(n-1)}{2}}} \sqrt{\prod_{i} \frac{1}{\lambda_{i}^{n+1}}}
$$

The proceeding is similar for the Wasserstein metric.

$$
\left\langle R E^{\prime}{ }_{i, i} R^{-1}, R E^{\prime}{ }_{j, j} R^{-1}\right\rangle_{W}^{W a s s e r s t e i n}=\lambda_{i},
$$

and for $i \neq j$ and $k \neq l$ we have:

$$
\left\langle R E^{\prime}{ }_{i, j} R^{-1}, R E^{\prime}{ }_{k, l} R^{-1}\right\rangle_{W}^{W a s s e r s t e i n}=\frac{\lambda_{i}+\lambda_{j}}{2},
$$

finally for $j \neq k$ :

$$
\left\langle R E^{\prime}{ }_{i, i} R^{-1}, R E^{\prime}{ }_{j, k} R^{-1}\right\rangle_{W}^{W a s s e r s t e i n}=0
$$

Then we can write: 


$$
G_{W}^{\text {Wasserstein }}=\left(\begin{array}{cccc}
\lambda_{1} & 0 & \cdot & \\
0 & \cdot & & \\
\cdot & & \lambda_{n} & \\
& & & \frac{\lambda_{i}+\lambda_{j}}{2} \\
& & &
\end{array}\right)
$$

finally we obtain

$$
\sqrt{\operatorname{det}\left(G_{W}^{W a s s e r s t e i n}\right)}=\frac{1}{2^{\frac{n(n-1)}{2}}} \sqrt{\prod_{i} \lambda_{i} \prod_{i<j}\left(\lambda_{i}+\lambda_{j}\right)}
$$

\section{B A measure on $\operatorname{SPD}(n)$ invariant under the action of rotations is a product between a measure on eigenvalues and the Haar measure of rotations}

Let us set the context. We are working on the space of positive definite matrices which have distinct eigenvalues. We will call this space $E$. The rotation matrices $\left(O^{+}\right)$induce a group action on $E$ :

$$
\begin{aligned}
O^{+} \times E & \longmapsto E \\
(R, M) & \longmapsto R M R^{t}
\end{aligned}
$$

For every matrix $M$ of $E$, there exist $R \in O^{+}$such that $R M R^{t}=\left(\begin{array}{ccc}\lambda_{1} & 0 & 0 \\ 0 & \lambda_{2} & 0 \\ 0 & 0 & \lambda_{3}\end{array}\right)$, and as such, we will note the space of diagonal matrices with positive strictly increasing eigenvalues as $E / O^{+}$. Furthermore, the following map

$$
\begin{gathered}
\phi: E / O^{+} \times O^{+} \rightarrow E \\
\left(\left(\begin{array}{ccc}
\lambda_{1} & 0 & 0 \\
0 & \lambda_{2} & 0 \\
0 & 0 & \lambda_{3}
\end{array}\right), R\right) \longmapsto R\left(\begin{array}{ccc}
\lambda_{1} & 0 & 0 \\
0 & \lambda_{2} & 0 \\
0 & 0 & \lambda_{3}
\end{array}\right) R^{T}
\end{gathered}
$$

is a diffeomorphism. For a matrix $M$ in $E$ we denote $M_{E / O^{+}}$and $M_{O^{+}}$the elements of $E / O^{+}$and $O^{+}$such that

$$
M=M_{O^{+}} M_{E / O^{+}} M_{O^{+}}^{T}
$$


We seek to show that any measure $\mu$ (with a density with respect to the Lebesgue measure) on $E$ invariant by the action of $O^{+}$can be written as a product measure on $E / \mathrm{O}^{+} \times \mathrm{O}^{+}$, where the measure on $\mathrm{O}^{+}$is the Haar measure $\mu_{\mathrm{O}^{+}}$(the unique measure on $\mathrm{O}^{+}$that is invariant by translations).

Consider a measurable subset $A$ of $E / O^{+}$and let $\mu_{A}$ be the measure on $\mathrm{O}^{+}$defined by

$$
\mu_{A}(B)=\int_{E} \mathbf{1}_{A}\left(M_{E / O^{+}}\right) \mathbf{1}_{B}\left(M_{O^{+}}\right) d \mu(M)
$$

We are going now show that this measure on $O^{+}$is translation invariant, and consequently a multiple of the Haar measure on $\mathrm{O}^{+}$.

$$
\begin{aligned}
\mu_{A}\left(R B R^{T}\right) & =\int_{E} \mathbf{1}_{A}\left(M_{E / O^{+}}\right) \mathbf{1}_{R B R^{T}}\left(M_{O^{+}}\right) d \mu(M) \\
& =\int_{E} \mathbf{1}_{A}\left(M_{E / O^{+}}\right) \mathbf{1}_{B}\left(R^{T} M_{O^{+}} R\right) d \mu(M) \\
& =\int_{E} \mathbf{1}_{A}\left(\left(R^{T} M R\right)_{E / O^{+}}\right) \mathbf{1}_{B}\left(\left(R^{T} M R\right)_{O^{+}}\right) d \mu(M),
\end{aligned}
$$

by the invariance of $\mu$, we obtain

$$
\begin{aligned}
\mu_{A}\left(R B R^{T}\right) & =\int_{E} \mathbf{1}_{A}\left(M_{E / O^{+}}\right) \mathbf{1}_{B}\left(M_{O^{+}}\right) d \mu(M) \\
& =\mu_{A}(B)
\end{aligned}
$$

Therefore, there exists $\lambda(A) \in \mathbb{R}$ such that $\mu_{A}=\lambda(A) \mu_{O^{+}}$. If $A, B$ are in $\mathrm{E} / \mathrm{O}^{+} \times \mathrm{O}^{+}$, then

$$
\begin{aligned}
\mu(\phi(A \times B)) & =\int_{E} \mathbf{1}_{A}\left(M_{E / O^{+}}\right) \mathbf{1}_{B}\left(M_{O^{+}}\right) d \mu(M) \\
& =\mu_{A}(B) \\
& =\lambda(A) \mu_{O^{+}}(B) .
\end{aligned}
$$

Furthermore, it is easy to see that $A \mapsto \lambda(A)$ is a measure on $E / O^{+}$.

Since the sets $A \times B$ generate the sets of measurable subsets of $E / O^{+} \times O^{+}$ (according to Dynkin's Theorem), the image of $\mu$ by $\phi$ is the product of the two measures $\lambda$ and $\mu_{O^{+}}$on $A$ and $B$ respectively.

It is also worth noting that $\lambda$ has a density with respect to the Lebesgue measure. 